ARTICLE

\title{
Perovskite multifunctional logic gates via bipolar photoresponse of single photodetector
}

\author{
Woochul Kim (1) 1,2,7, Hyeonghun Kim (10) 2,3,7, Tae Jin Yoo4 , Jun Young Lee ${ }^{1,5}$, Ji Young Jo (1) 1, Byoung Hun Lee ${ }^{4}$,
} Assa Aravindh Sasikala (i) ${ }^{6}$, Gun Young Jung (i) ${ }^{1 凶} \&$ Yusin Pak (i) ${ }^{2 凶}$

The explosive demand for a wide range of data processing has sparked interest towards a new logic gate platform as the existing electronic logic gates face limitations in accurate and fast computing. Accordingly, optoelectronic logic gates (OELGs) using photodiodes are of significant interest due to their broad bandwidth and fast data transmission, but complex configuration, power consumption, and low reliability issues are still inherent in these systems. Herein, we present a novel all-in-one OELG based on the bipolar spectral photoresponse characteristics of a self-powered perovskite photodetector (SPPD) having a backto-back $p^{+}-i-n-p-p^{+}$diode structure. Five representative logic gates ("AND", "OR", "NAND", "NOR", and "NOT") are demonstrated with only a single SPPD via the photocurrent polarity control. For practical applications, we propose a universal OELG platform of integrated $8 \times 8$ SPPD pixels, demonstrating the $100 \%$ accuracy in five logic gate operations irrelevant to current variation between pixels.

\footnotetext{
${ }^{1}$ School of Materials Science and Engineering (SMSE), Gwangju Institute of Science and Technology (GIST), Gwangju 61005, Republic of Korea. ${ }^{2}$ Sensor System Research Center, Korea Institute of Science and Technology (KIST), Seoul 02792, Republic of Korea. ${ }^{3}$ School of Engineering Technology, Purdue University, West Lafayette, IN 47907, USA. ${ }^{4}$ Department of Electrical Engineering, Pohang University of Science and Technology, Gyeongbuk 37673, Republic of Korea. ${ }^{5}$ Electronic Materials Research Center, Korea Institute of Science and Technology (KIST), Seoul 02792, Republic of Korea. ${ }^{6}$ Nano and Molecular Systems Research Unit (NANOMO), University of Oulu, Oulu 90750, Finland. ${ }^{7}$ These authors contributed equally: Woochul Kim, Hyeonghun Kim.

凶email: gyjung@gist.ac.kr; yusinpak@kist.re.kr
} 
O ptoelectronic logic gates (OELGs) are receiving significant attention as crucial building block of future integrated circuits for accurate and fast data processing ${ }^{1-4}$. Existing circuits or processors based on electronic logic gates would face limitations in computing extensive data sets which are expected to markedly increase in the fourth industrial revolution age, due to performance shortfalls in switching, operation, computing, and decision making/regenerating ${ }^{5,6}$. Thus, developing an innovative logic gate platform that can implement faster computation with less power consumption is imperative to fulfill upcoming new computing trends.

Accordingly, all-optical logic gates using light inputs and outputs have been actively studied to replace electronic binary adders, binary counters, decision circuits, optical processors, data encoders, and bit-pattern recognition circuits ${ }^{7}$. These optical logic gates are constructed based on intricate designs of optical components such as terahertz asymmetric demultiplexers, nonlinear interferometers, and semiconductor amplifiers ${ }^{8,9}$. Although the optical logic gate system has attractive merits, including good optical gain, saturation output power, and gain bandwidth, practical applications have yet to be reported due to its expensive and large components ${ }^{10}$. Moreover, the straight propagation of light is a considerable obstacle for building a highly integrated system since placing optical components in an integrated manner, i.e., the arrangement with many optical curvatures may cause a sizeable optical loss ${ }^{11}$.

Therefore, an OELG, which has recently been in the spotlight due to its broad bandwidth, fast data transmission, and low $\operatorname{cost}^{12,13}$, is considered a good candidate for replacing the existing logic gates (e.g., electronic and all-optical). A typical OELG consists of more than two semiconductor junctions that interact with the light inputs and release a Boolean electrical output ${ }^{14,15}$. The most well-studied OELGs are $\mathrm{p}-\mathrm{n}$ heterojunction photodiodes or photodetectors (PDs) such as $\mathrm{ZnO} / \mathrm{CdSe}^{16}, \mathrm{CuInS}_{2} /$ $\mathrm{TiO}_{2}{ }^{17}$, and $\mathrm{n}-\mathrm{Si} / \mathrm{MoO}_{3-x}{ }^{18}$. Since these PDs operate mainly based on unidirectional carrier transport, most studies have only reported a single logic gate (e.g., AND or OR) to the best of our knowledge. Thus, to implement multiple logic functions, there is a strong need to develop a layered semiconducting PD platform that can freely control the direction of photocarrier transport. For instance, Yang et al. reported the wavelength-induced dualpolarity phenomenon from a $\mathrm{p}-\mathrm{n}$ heterojunction diode composed of n-type $\mathrm{ZnO}$ nanowires and p-type thermoelectric thin film $\left(\mathrm{SnS} \text { or } \mathrm{Sb}_{2} \mathrm{Se}_{3}\right)^{19-22}$. In addition, particular circuit configurations are required for operating each logic function, and the reliability of output discrimination is strongly influenced by electrical noise or output variation of each logic gate $14,23,24$. Therefore, the difficulty in integration, high power consumption, and inaccurate signal characteristics should be enhanced to employ them in practical OELG applications.

With the recent success of perovskite photovoltaics, many studies have focused on perovskite optical sensors and PDs. The crucial challenge of these devices is the achievement of high gain, low power, and miniaturization. In this regard, organometal halide perovskite PDs allowing high absorption coefficient ${ }^{25}$, excellent quantum efficiency ${ }^{26,27}$, and broad spectral responsivity ${ }^{28-30}$ can be promising as the OELG material and device. The superior optoelectronic properties of organometal halide perovskite have been demonstrated in many studies ${ }^{31-35}$. Although high-speed broadband detection and superior responsivity characteristics have been developed, such perovskite PD platforms operating on the principle of unidirectional carrier transport cannot be used as an integrated OELG component that performs multiple logic functions.

This paper reports a novel all-in-one OELG system based on the bipolar spectral photoresponse characteristics of a self-powered perovskite photodetector (SPPD). Five representative logics (“AND", "OR", "NAND”, "NOR", and "NOT”) were demonstrated with only a single SPPD, which converts the optical inputs to electrical outputs. The pivotal keys for executing the multiple Boolean logics are: (1) a "back-to-back configuration" (i.e., $\mathrm{p}^{+}-\mathrm{i}-\mathrm{n}-\mathrm{p}-\mathrm{p}^{+}$), based on two vertically stacked perovskite diodes; and (2) an "optical gate modulation" using visible and near-infrared light. The back-to-back SPPD demonstrated a sensitive photoresponse and a substantial on-off ratio. An eightby-eight $(8 \times 8)$ logic array consisting of 64 SPPD pixels successfully executed the five basic logic gates at $100 \%$ accuracy, insusceptible to electrical noise or current variation between the pixels owing to bipolar photoresponse.

\section{Results}

Back-to-back $\mathbf{p}^{+}$-i-n-p-p $\mathbf{p}^{+}$structure of the SPPD. The SPPD was composed of vertically stacked low-band perovskite $\left(\mathrm{FA}_{0.5} \mathrm{MA}_{0.5} \mathrm{~Pb}_{0.4} \mathrm{Sn}_{0.6} \mathrm{I}_{3}\right.$, near infrared-Perov (NIR-Perov)) and high-band perovskite ( $\mathrm{MAPbI}_{3}$, visible-Perov (Vis-Perov)), as depicted in Fig. 1a. Phenyl- $\mathrm{C}_{61}$-butyric acid methyl ester (PCBM) layer was inserted as an n-type semiconductor between Vis-Perov and NIR-Perov, as shown in the cross-sectional transmission electron microscope (TEM) image. The perovskite layers were prepared by a two-step method: (i) vapor-phase thermal evaporation of a metal halide film $\left(\mathrm{PbI}_{2}\right.$ for $\mathrm{MAPbI}_{3}$ and $\mathrm{PbI}_{2} / \mathrm{SnI}_{2}$ for $\mathrm{FA}_{0.5} \mathrm{MA}_{0.5} \mathrm{~Pb}_{0.4} \mathrm{Sn}_{0.6} \mathrm{I}_{3}$ ); and (ii) conversion step to form the perovskite phase (see more details in "Methods"). The metal halide films formed by the two-step method were so resistive to solvent infiltration that the underlying layers were not chemically damaged, as reported in previous studies ${ }^{36,37}$. The ratio between organic and metallic cations $\left(\mathrm{FA}^{+}\right.$(formamidinium): $\mathrm{MA}^{+}$ $($ methylammonium $) \approx 1: 1$ and $\mathrm{Pb}^{2+}: \mathrm{Sn}^{2+} \approx 4: 6$ ) for the low-band NIR-Perov was optimally determined to have high film crystallinity, efficient device performance, and narrow bandgap (Supplementary Fig. 1) ${ }^{38,39}$. The p-type PEDOT:PSS and SpiroOMeTAD were used as bottom and top hole-transport layers connected to ITO and Au electrodes, respectively. The thickness of each perovskite layer $(300 \mathrm{~nm}$ NIR-Perov and $200 \mathrm{~nm}$ VisPerov) was decided via a theoretical simulation, aiming a photoconductive gain ratio $\left(G_{940} / G_{625}\right)$ of 1 for efficient current offset, which is beneficial for the tuning of the logic gate operation (Supplementary Fig. 2). All the layers were vertically stacked without notable wrinkles and defects at each interface.

The element distribution was investigated by scanning transmission electron microscopy (STEM) equipped with energy-dispersive X-ray spectroscopy (EDX), as shown in Fig. 1b. $\mathrm{Pb}$ (red) and I (pink) elements were distributed uniformly in both perovskite layers (NIR-Perov and Vis-Perov). Sn (green) was only observed within the NIR-Perov layer, confirming that no perovskite merging occurred between the layers. Wide X-ray photoelectron spectroscopy (XPS) demonstrated the atomic ratio of $\mathrm{Pb}$ and $\mathrm{Sn}$ in the NIR-Perov layer to be approximately $4: 6$, which is known as the ideal composition for NIR absorption (Supplementary Fig. 3). In the perovskite phase, $\mathrm{Sn}^{2+}$ and $\mathrm{Pb}^{2+}$ were the primary oxidation states, according to the narrow $\mathrm{Sn} 3 d$ and $\mathrm{Pb} 4 f$ XPS spectra (Supplementary Fig. 2b and c), implying the superior film quality of NIR-Perov layer without any oxidation of $\mathrm{Sn}^{2+}$. Notably, the absence of a $\mathrm{Sn}^{4+}$ peak substantiated that a thiourea additive (see "Methods") was an effective anti-oxidative agent for protecting $\mathrm{Sn}^{2+}$ cations from the undesirable oxidation ${ }^{40}$. The results of UV-Vis-NIR spectroscopy (Fig. 1c) showed that the Vis-Perov layer $\left(\mathrm{MAPbI}_{3}\right)$ absorbed the entire visible light $(400-750 \mathrm{~nm})$ while the NIR-Perov layer $\left(\mathrm{FA}_{0.5} \mathrm{MA}_{0.5} \mathrm{~Pb}_{0.4} \mathrm{Sn}_{0.6} \mathrm{I}_{3}\right)$ could absorb even the infrared light $(400-1000 \mathrm{~nm})$. The bandgaps of both perovskites were 1.67 and 
a

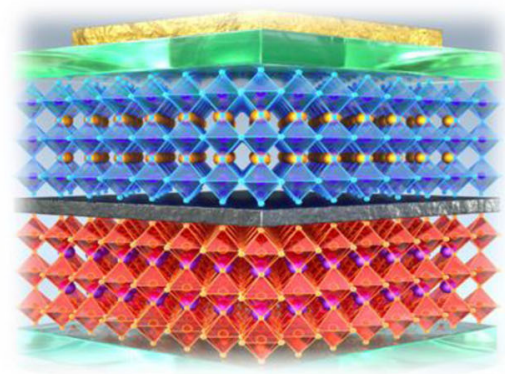

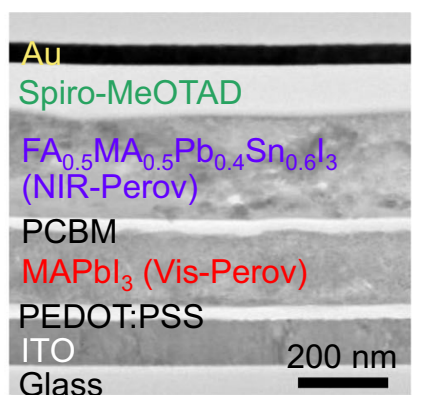

Glass

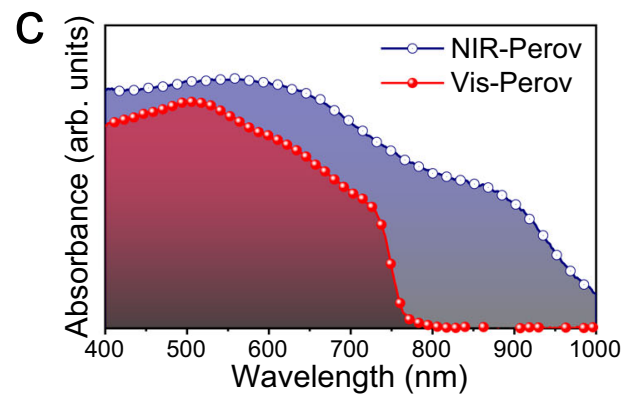

d
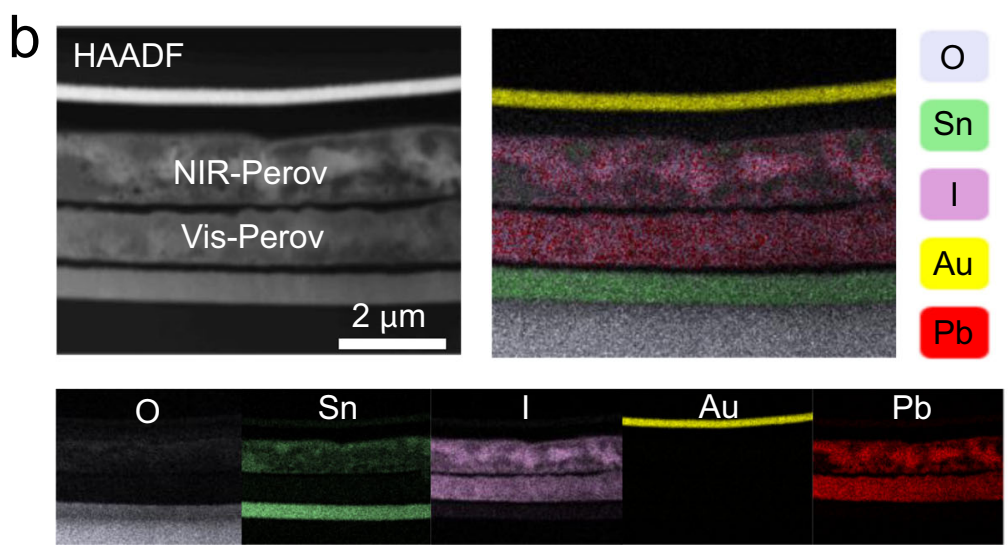
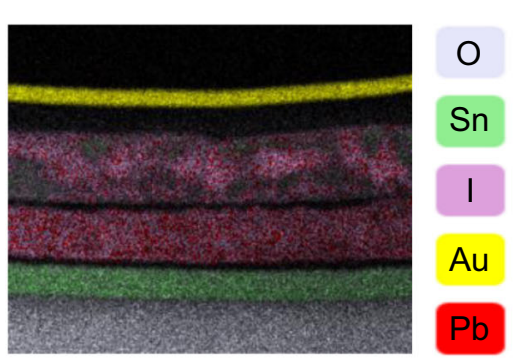

$\mathrm{Au}$
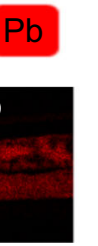

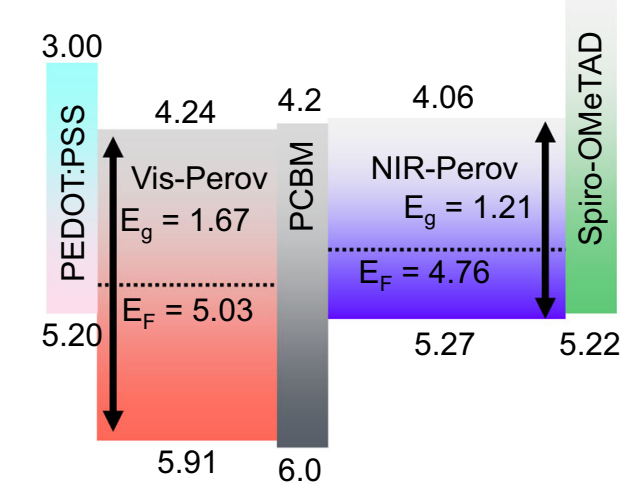

Fig. 1 Characterization of the back-to-back SPPD. a Schematic of vertically stacked SPPD and its corresponding cross-sectional TEM image. b STEM-

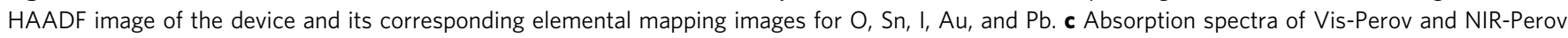

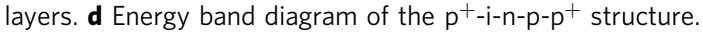

$1.21 \mathrm{eV}$ for the Vis-Perov and NIR-Perov layers, respectively, as calculated by the Tauc-plot method (Supplementary Fig. 4).

Figure $1 \mathrm{~d}$ depicts the energy band diagram of SPPD, which was determined based on the result of UV photoelectron spectroscopy (UPS) (Supplementary Fig. 5) and relevant studies (for heavily doped p-type PEDOT:PSS ${ }^{41}$, Spiro-OMeTAD ${ }^{42}$, and n-type $\mathrm{PCBM}^{41}$ ). The Vis-Perov layer was intrinsic (i-type), and the NIR-Perov layer was lightly doped p-type, which were additionally supported by Hall measurements (Supplementary Table 1).

The PCBM could transfer electrons to either Vis-Perov or NIRPerov layer at each heterojunction due to a low interfacial energy barrier. Density functional theory (DFT) calculations were conducted to obtain insight into the charge transport through the PCBM bridge (Supplementary Note 1). We found that additional density of states (DOS) below the conduction band were existing at the interface between perovskites and PCBM layers, which further facilitates the electron transfer to both perovskite layers (Supplementary Figs. 6 and 7). The symmetric band structure (back-to-back $\mathrm{p}^{+}-\mathrm{i}-\mathrm{n}-\mathrm{p}-\mathrm{p}^{+}$configuration) originating from the combination of two perovskite diodes provided a critical working principle that could control charge transfer in both directions (i.e., a bipolar spectral photoresponse) in response to irradiation conditions (e.g., wavelength and power density).

Bipolar optoelectronic properties of the SPPD. The back-toback SPPD $\left(\mathrm{p}^{+}-\mathrm{i}-\mathrm{n}-\mathrm{p}-\mathrm{p}^{+}\right)$showed bipolar spectral photoresponse depending on irradiation condition, which was quite different from a single diode (e.g., p-i-n or pn structure), yielding only unipolar photoresponse. The SPPD generated currents with opposite polarities depending on the visible $(530 \mathrm{~nm})$ and NIR $(940 \mathrm{~nm})$ irradiation, as shown in Fig. 2a. Open circuit voltages $\left(V_{\text {OC }}\right)$ were $-0.22 \mathrm{~V}$ for $530 \mathrm{~nm}$ and $+0.09 \mathrm{~V}$ for $940 \mathrm{~nm}$ at a power density of $1 \mathrm{~mW} \mathrm{~cm}^{-2}$. Figure $2 \mathrm{~b}$ exhibits three on/off cycles of SPPD at zero voltage. The responsivity values for the positive and negative photocurrents were $5.8 \mathrm{~mA} \mathrm{~W}^{-1}$ for $530 \mathrm{~nm}$ and $8.7 \mathrm{~mA} \mathrm{~W}^{-1}$ for $940 \mathrm{~nm}$ irradiation (Fig. 2b). The rise/decay times of SPPD with an active area of $0.04 \mathrm{~mm}^{2}$ were $62 / 680 \mu$ s for $530 \mathrm{~nm}$ and $40 / 72 \mu$ s for $940 \mathrm{~nm}$ (Supplementary Fig. 8). Even though there is still an apparent gap compared to the state-of-art level (e.g., hundreds of ns, Supplementary Table 2), it can be enhanced using a high-crystalline perovskite film with further downsizing of the active area.

The bipolar spectral photoresponse of SPPD was investigated by illumination from 400 to $1000 \mathrm{~nm}$ (Fig. 2c). The responsivity was maximum at $500 \mathrm{~nm}$, which was gradually decreasing with increasing the wavelength, and became zero at $\sim 750 \mathrm{~nm}$. Beyond $750 \mathrm{~nm}$, the responsivity dramatically increased with opposite polarity. The zero responsivity point $(750 \mathrm{~nm})$ can be varied with the perovskite and intermediate components. Based on the spectral responsivity results, photoconductive gain $\left(G_{\lambda}\right)$ and detectivity $\left(D_{\lambda}\right)$ values were calculated as shown in Supplementary Fig. 9. The $G_{\lambda}$ values were 0.02 and 0.008 for 530 and $940 \mathrm{~nm}$ light illumination, respectively. The corresponding $D_{\lambda}$ values were calculated as 1.89 and $1.27 \times 10^{11}$ Jones, respectively.

The SPPD retained its initial responsivity for 3000 pulses $(5 \mathrm{~s}$ on/5 s off) under ambient condition as shown in Supplementary Fig. 10. For long-term stability testing, the SPPD was stored in dry-air condition for 1 year. The saturated on-current was reduced to $90 \%$ and $50 \%$ of the initial value under 530 and $940 \mathrm{~nm}$, respectively (Supplementary Fig. 11). The relatively significant reduction under the NIR light can be attributed to the instability of $\mathrm{Sn}$ cations in the air ${ }^{42,43}$.

To theoretically evidence the bipolar spectral photoresponse, we performed an optical field simulation. A charge generation rate $\left(C_{\mathrm{R}}\right)$, occurring along a coronal plane of the back-to-back structure, was simulated under monochromatic irradiation 

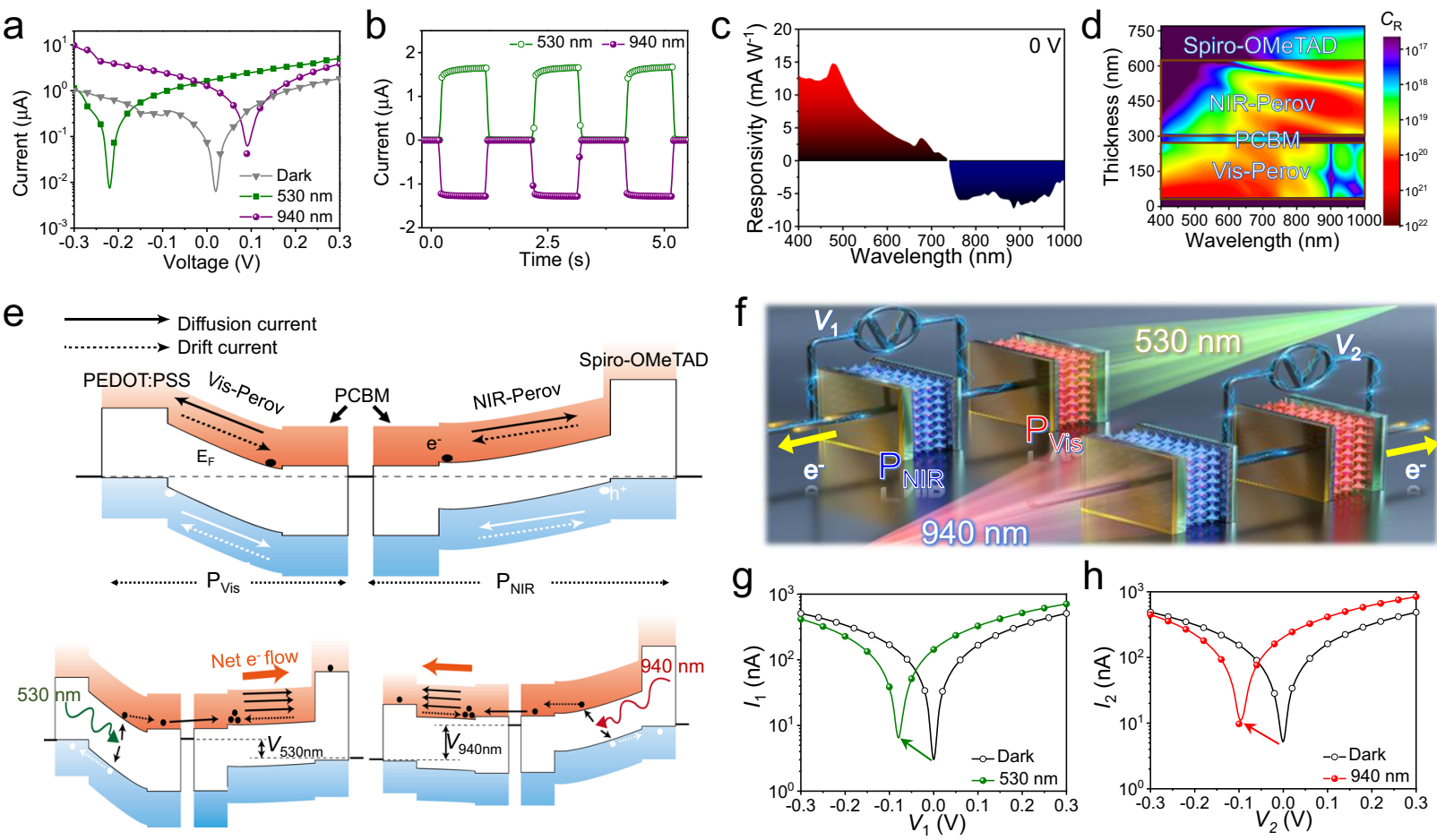

Fig. 2 Optoelectronic properties of the SPPD. a, b $I-V$ curves and steady-state currents at zero bias under dark and irradiation conditions (530 and $940 \mathrm{~nm}$ lights at $1 \mathrm{~mW} \mathrm{~cm}^{-2}$ ). c Bipolar spectral photoresponse of the SPPD as a function of wavelength from 400 to $1000 \mathrm{~nm}$ at a $10 \mathrm{~nm}$ interval. d Charge generation rate $\left(C_{R}\right)$ distribution within the $p^{+}-i-n-p-p^{+}$structure vs. wavelength of incident light. e Energy band diagrams of the SPPD ( $P_{V i s}$ and $\mathrm{P}_{\mathrm{NIR}}$ serially connected through PCBM); under equilibrium state (top), $530 \mathrm{~nm}$ light irradiation onto the $\mathrm{P}_{\mathrm{Vis}}$ (bottom-left), and $940 \mathrm{~nm}$ light irradiation onto the $P_{N I R}$ (bottom-right). $\mathbf{f}$-h Experiments for substantiating the working mechanism of (e). $\mathbf{f}$ Schematic illustration of equivalent circuits for the separate perovskite diodes ( $P_{\text {Vis }}$ and $P_{N I R}$ ) connected with an electrical wire. $I-V$ curves of $\mathbf{g} P_{\text {NIR }}$ in response to the $530 \mathrm{~nm}$ light irradiation onto the $P_{\text {Vis }}$ and $\mathbf{h} \mathrm{P}_{\text {Vis }}$ in response to the $940 \mathrm{~nm}$ light irradiation onto the $\mathrm{P}_{\mathrm{NIR}}$.

$(400-1000 \mathrm{~nm})$. The $C_{\mathrm{R}}$ was calculated as follows:

$$
C_{\mathrm{R}}=\frac{2 \pi \varepsilon_{0} n k}{h}\|\mathbf{E}\|^{2}
$$

where $\varepsilon_{0}$ is the permittivity of free space, $h$ is the Planck constant, $n$ and $k$ are the real and imaginary parts of the refractive index, and $\mathbf{E}$ is the optical field. The optical field was calculated based on the transfer matrix method ${ }^{44}$ (Supplementary Fig. 12) using complex refractive indices and film thicknesses of components (Supplementary Fig. 13). Figure 2d exhibits the distribution profile of $C_{\mathrm{R}}$ as a function of the wavelength. The $C_{\mathrm{R}}$ intensities of two perovskites (NIR-Perov and Vis-Perov) at around $750 \mathrm{~nm}$ were approximately equivalent such that they readily offset each other, resulting in the net-zero photocurrent coinciding with Fig. 2c. The Vis-Perov layer demonstrated an intense (red) $C_{R}$ field in the visible range $(400-750 \mathrm{~nm}$ ), which weakened (blue) beyond $750 \mathrm{~nm}$. In contrast, the $C_{\mathrm{R}}$ field in the NIR-Perov layer strengthened from $750 \mathrm{~nm}$. The main advantage of back-to-back configuration was that the offset wavelength could be readily tuned by diode materials and stacking order of perovskite materials, implying versatile applications in future optical and optoelectronic sensor chips.

The energy band diagram of SPPD could be represented as two separate diodes connected in series: $\mathrm{p}^{+}-\mathrm{i}-\mathrm{n}\left(\mathrm{P}_{\mathrm{Vis}}\right.$ : PEDOT:PSS/ Vis-Perov/PCBM); and n-p-p ${ }^{+}$( $\mathrm{P}_{\text {NIR }}$ : PCBM/NIR-Perov/SpiroOMeTAD) as depicted in the upper scheme of Fig. 2e. The large difference in Fermi levels between the n-type PCBM and two heavily doped p-type layers (PEDOT:PSS and Spiro-OMeTAD) produced built-in potentials that attracted charges to the PCBM. More details on the built-in potential and energy band-bending are explicated in Supplementary Fig. 14. Without external stimuli (e.g., light or electrical bias), the diffusion current driven by the carrier concentration gradient and the drift current by the potential gradient were equivalent, resulting in a net-zero current. However, as shown in the lower scheme of Fig. 2e, the balance between the diffusion and drift current was broken under illumination. Under $530 \mathrm{~nm}$, the electrons generated at the VisPerov $\left(\mathrm{P}_{\mathrm{Vis}}\right)$ layer diffused and piled up at the interface between PCBM and NIR-Perov $\left(\mathrm{P}_{\mathrm{NIR}}\right)$ layer. When enough electrons were accumulated at the interface, an electrical potential $\left(V_{530 \mathrm{~nm}}\right)$ was built across the $\mathrm{P}_{\mathrm{NIR}}$ which cancel out the inherent built-in potential, thus generating a net electron diffusion toward the Spiro-OMeTAD layer without an external bias. Likewise, under $940 \mathrm{~nm}$, the increased electron concentration gradient induced a potential $\left(V_{940 \mathrm{~nm}}\right)$ across Vis-Perov layer, leading to a net photocurrent. It is noteworthy that when the PCBM layer was not inserted, the photocurrent flowed unidirectionally from VisPerov to NIR-Perov as shown in Supplementary Fig. 15.

To experimentally prove the aforementioned optoelectronic mechanism that a current (or potential) is generated in a diode (not illuminated) by illuminating the other electrically connected diode, we fabricated a series circuit consisting of two separate perovskite diodes $\left(\mathrm{P}_{\mathrm{Vis}}\right.$ and $\left.\mathrm{P}_{\mathrm{NIR}}\right)$, as depicted in Fig. 2f. Only one diode was irradiated with photo-active light, while the other was deliberately blocked from it. As shown in Fig. $2 \mathrm{~g}$, for the $530 \mathrm{~nm}$ irradiation onto the $\mathrm{P}_{\mathrm{Vis}}$, the $\mathrm{P}_{\mathrm{NIR}}$ produced a significant current of $\sim 110 \mathrm{nA}$ at $0 \mathrm{~V}$ owing to a generated electrical potential $\left(V_{\mathrm{OC}}\right.$ shift of $-0.08 \mathrm{~V})$. Similarly, under $940 \mathrm{~nm}$ irradiation onto the $\mathrm{P}_{\mathrm{NIR}}$, the $V_{\mathrm{OC}}$ of $\mathrm{P}_{\mathrm{Vis}}$ shifted $(-0.1 \mathrm{~V})$, producing a reverse current of $\sim 120 \mathrm{nA}$ (Fig. 2h). These results demonstrated the self-powered SPPD and the necessity of integrated back-to-back SPPD ( ${ }^{+}-\mathrm{i}-\mathrm{n}-$ $\mathrm{p}-\mathrm{p}^{+}$) for enabling the bipolar spectral photoresponse. 

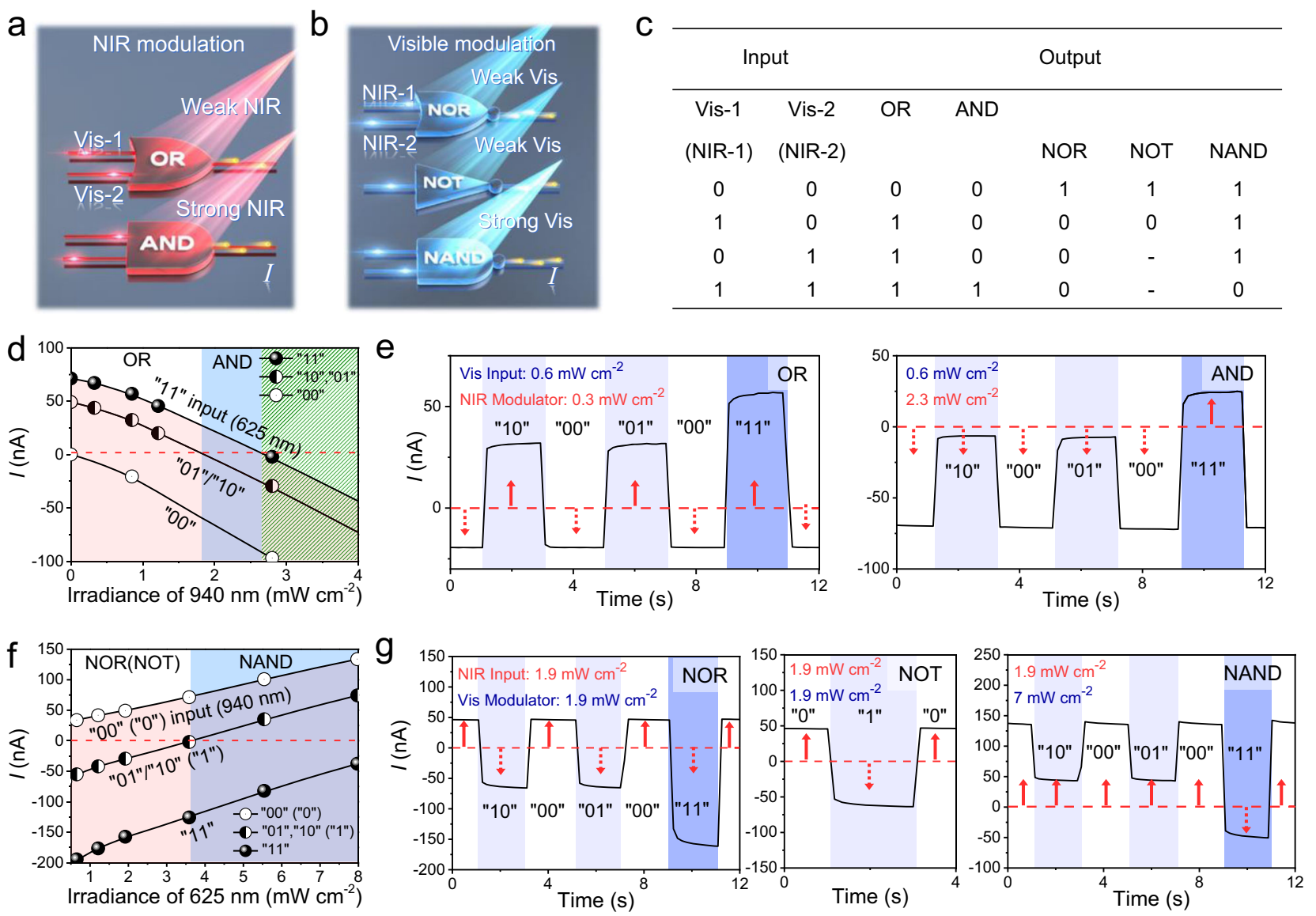

Fig. 3 Demonstration of the OELGs via the single SPPD. a, b Symbolic schematics of five OELGs executed with single SPPD. a "OR" and "AND" gates using two visible light inputs $(625 \mathrm{~nm})$ under NIR light $(940 \mathrm{~nm})$ gate modulations; b "NOR", "NOT", and "NAND" gates using NIR light inputs (940 nm) under visible light $(625 \mathrm{~nm})$ gate modulations. I is an output photocurrent. c Truth table of the five OELGs for the four input combinations. d-g Optoelectronic logic gate operations. d Photocurrent output via visible light ( $625 \mathrm{~nm})$ inputs depending on NIR irradiance (940 nm). e Transient photocurrent curves of "OR" and "AND" gates in response to visible inputs $\left(0.6 \mathrm{~mW} \mathrm{~cm}^{-2}\right)$ under NIR modulation of 0.3 and $2.3 \mathrm{~mW} \mathrm{~cm}^{-2}$, respectively. f Photocurrent output via NIR $(940 \mathrm{~nm})$ light inputs depending on visible irradiance $(625 \mathrm{~nm})$. g Transient photocurrent curves of "NOR", "NOT", and "NAND" gates in response to NIR inputs $\left(1.9 \mathrm{~mW} \mathrm{~cm}^{-2}\right)$ under visible modulation of $1.9,1.9$, and $7 \mathrm{~mW} \mathrm{~cm}^{-2}$, respectively.

Demonstration of five basic OELGs. Based on the bipolar spectral photoresponse of SPPD, we propose a novel OELG system that can execute five basic logics of "OR", "AND", "NAND", "NOR", and "NOT" with only single SPPD (Fig. 3a, b). The key factor for tuning logic functions in this system is the "optical gate modulator", which involves irradiation using different wavelengths and intensities. Delicate tuning of logic functions via the light intensity modulation for the opposite photocurrent-offset can be most efficient when the two light wavelengths have similar photoconductive gains. As the $625 \mathrm{~nm}$ light revealed a wellmatched photoconductive gain (0.007) with that (0.008) in case of the $940 \mathrm{~nm}$ light (Supplementary Fig. 9a), hereafter, we utilized these two light sources for the following logic gate operations. Prior to the use of $625 \mathrm{~nm}$, we confirmed that the $625 \mathrm{~nm}$ irradiation generated the opposite photocurrents compared to the $940 \mathrm{~nm}$ case (Supplementary Fig. 16). Figure 3c shows the electrical logic outputs of the SPPD-OELG in response to the optical inputs (" 00 ", " 10 ", " 01 ", and " 11 "), in which " 0 " and " 1 " signify "on" and "off" light input state, respectively. To the best of our knowledge, this is the first study that has successfully demonstrated five basic logic gates with a single device (SPPD) without any change in the circuit configurations. To emulate the "AND" and "OR" gates (Fig. 3d), we investigated the photocurrents of SPPD in response to visible light (i.e., $625 \mathrm{~nm}$ at $0.6 \mathrm{~mW} \mathrm{~cm}^{-2}$ ) input combinations (“00", “ 01 ”, “ 10 ", and " 11 ”) under varying
NIR irradiances $(940 \mathrm{~nm})$. Here, the NIR light (gate modulator) was irradiated simultaneously with the visible light input, producing a negative offset photocurrent against the positive current by the visible light input. The red dashed line in Fig. $3 \mathrm{~d}$ represents the fiducial level ( $0 \mathrm{nA})$, which set the output state as " 1 " and " 0 " for the values above and below it, respectively.

Under low NIR irradiance $\left(<1.8 \mathrm{~mW} \mathrm{~cm}^{-2}\right)$, if either input was true ("01" or " 10 "), the output photocurrent always exceeded the fiducial level positively. The negative photocurrent below $0 \mathrm{nA}$ was obtained only for the " 00 " input, executing the "OR" gate. Meanwhile, under the NIR irradiance from 1.8 to $2.6 \mathrm{~mW} \mathrm{~cm}^{-2}$, the "AND" gate was implemented to have " 1 " output state only when both inputs were true (" 11 "). The current levels of " 10 " and " 01 " inputs completely dropped below $0 \mathrm{nA}$ by the NIR-modulated negative offset photocurrent. The transient curves (Fig. 3e) show all the possible input scenarios for both the "OR" and "AND" gates. The upward and downward arrows represent the " 1 " and " 0 " output states, respectively. For the "OR" gate under the NIR irradiance of $0.3 \mathrm{~mW} \mathrm{~cm}^{-2}$, all the input combinations except for " 00 " input induced a positive photocurrent above $25 \mathrm{nA}$, corresponding to " 1 " output state. Interestingly, the "OR" gate promptly was transformed into the "AND" gate when the NIR irradiance increased to $2.3 \mathrm{~mW} \mathrm{~cm}^{-2}$, which produced a positive photocurrent only for the "11" input. This tuning technique (OR $\leftrightarrow$ AND) using the NIR modulation was reversible and repeatable. 
Furthermore, the unique spectral photoresponse of SPPD also allowed to execute inverting logic gates, such as "NAND", "NOR", and "NOT". Figure $3 \mathrm{f}$ shows the photocurrents of SPPD under NIR inputs (i.e., $940 \mathrm{~nm}$ at $1.9 \mathrm{~mW} \mathrm{~cm}^{-2}$ ) as a function of the visible irradiance $(625 \mathrm{~nm})$. In contrast to the previous NIR gate modulation, the photocurrent was increased with the irradiance of visible modulation. Under low visible irradiance $\left(<3.6 \mathrm{~mW} \mathrm{~cm}^{-2}\right)$, the photocurrents were smaller than the fiducial level ( 0 nA) if one ("10" or "01") or both ("11") NIR inputs were applied. However, in case of " 00 " NIR input, a positive photocurrent was generated, corresponding to either the "NOR" or "NOT" logic gate. On the other hand, as the visible irradiance increased above $3.6 \mathrm{~mW} \mathrm{~cm}-2$, the photocurrent was positively converted except for the case of "11" NIR inputs, operating the "NAND" gate.

Figure $3 \mathrm{~g}$ shows the OELG operation for implementing the three inverting logic gates. Visible modulation irradiances of 1.9 and $7 \mathrm{~mW} \mathrm{~cm}{ }^{-2}$ were used for "NOR" (and "NOT") and "NAND", respectively. Under a visible irradiance of $1.9 \mathrm{~mW} \mathrm{~cm}^{-2}$, the output state of " 1 " ( $46 \mathrm{nA})$ was obtained only for the " 00 " NIR input (-64 nA for the " 01 " and " 10 " inputs and $-160 \mathrm{nA}$ for " 11 " input), demonstrating the "NOR" gate. In addition, at the same modulating condition, the " 0 " and " 1 " NIR single inputs revealed " 1 " (46 nA) and "0" (-64 nA) outputs, respectively, executing the "NOT" gate. Under $7 \mathrm{~mW} \mathrm{~cm}^{-2}$ irradiance, a negative photocurrent was produced only by the "11" input, executing the "NAND" gate. In addition, we also confirmed logic gate operations using a pair of 530 and $940 \mathrm{~nm}$ light sources after precisely adjusting the irradiance of $530 \mathrm{~nm}$ light (Supplementary Fig. 17).

SPPD array platform for advanced OELG applications. For real-world applications, it is imperative to construct integrated SPPD-OELG platform and investigate its accuracy to ensure the practical chip-level feasibility. Figure 4a shows an eight-by-eight $(8 \times 8)$ crossbar-type SPPD array. Seven stacked layers (PEDOT:PSS/NIR-Perov/PCBM/Vis-Perov/Spiro-OMeTAD/MoO ${ }_{3}$ ) were sandwiched between the top (transparent ITO) and bottom $\mathrm{Au}$ ) electrodes (see more details in "Methods"). The crossed area (dotted square) between the two electrodes served as a singlepixel logic gate with an active area of $200 \times 200 \mu \mathrm{m}^{2}$; 64 pixels were integrated in total (Fig. 4b). Three-dimensional bar charts in Fig. $4 c$ demonstrate all the outputs for the five logic gates obtained from the 64 pixels. The red and blue bars represent the positive and negative photocurrents, respectively, with reference to the fiducial level of $0 \mathrm{nA}$ (gray face). All the corresponding pixels yielded the outputs with $100 \%$ accuracy in all five logic gates, irrelevant to the calculated coefficient of photocurrent variation (=standard deviation/average, $0.36-0.66$ ) which affects the uniformity of output signals.

Even after downsizing the pixel to $50 \times 50 \mu \mathrm{m}^{2}$, the SPPD array succeeded in yielding all the logic gate operations with $100 \%$ accuracy. The photocurrent was reduced with decreasing the active pixel size, but the output polarity was maintained in every pixel (Supplementary Figs. 18-20). The superior output reliability of SPPD array platform was attributed to our back-to-back SPPD structure that distinguished true or false output state based on the polarity of photocurrent rather than the difference between the unipolar current and fiducial level, normally used in other conventional logic gate devices.

Notably, from a practical perspective, this approach is an advancement over the reported optical and optoelectronic logic gates regarding the on/off ratio (theoretically infinite in this work, Supplementary Table 3), which is the key factor for precise output decision. Furthermore, the back-to-back SPPD is advantageous to multi-logic functionality with a single device, circuit compactness, and high spatial efficiency, compared to all kind of logic gate devices.

\section{Discussion}

We reported all-in-one OELG based on the SPPD to execute multi-logic gates that can build advanced logic gate circuits or processors. The pivotal point is the back-to-back SPPD structure $\left(\mathrm{p}^{+}-\mathrm{i}-\mathrm{n}-\mathrm{p}-\mathrm{p}^{+}\right)$consisting of the low-band and high-band perovskites. The photocurrent polarity of SPPD could be deliberately tuned by optical gate modulation using visible $(625 \mathrm{~nm})$ and NIR $(940 \mathrm{~nm})$ lights. Thus, the output state of " 1 " or "0" was determined by the positive or negative output photocurrent, respectively. The single SPPD succeeded in configuring five logics: "AND", "OR", "NAND", "NOR", and "NOT". The output discrimination of SPPD-OELG was totally based on the polarity of photocurrent rather than the difference between unipolar photoresponse and fiducial level, improving the accuracy and reliability of logic gate device significantly irrelevant to current variation or electrical noise. The OELG array platform involving 64 SPPD pixels executed all the five logic gates at $100 \%$ yield without any errors.

The data processing under specific instructions (e.g., add, multiply, or count) could be implementable with a combinational circuit of multiple SPPD-OELGs in a single chip, which is much spatially and costly efficient compared to the conventional logic circuit based on electronic transistors, potentially advancing to future applications for optical computing, optical communication, and logic memory. In short-term view, this development can be applicable to light-fidelity (Li-Fi) transmission ${ }^{21}$, security circuits $^{45}$, and healthcare sensors ${ }^{46}$, utilizing the distinguished optoelectronic output states based on the photocurrent polarity.

\section{Methods}

Fabrication of the SPPD. For the preparation of $\mathrm{MAPbI}_{3}$, a $\mathrm{PbI}_{2}$ film was thermally deposited on the PEDOT:PSS-coated ITO substrate, and MAI solution $\left(50 \mathrm{mg} \mathrm{mL}^{-1}\right.$ in IPA) was spin-coated on the sample at a rate of $3500 \mathrm{rpm}$ for $45 \mathrm{~s}$. The $\mathrm{PbI}_{2}$ and MAI layers were converted into the $\mathrm{MAPbI}_{3}$ at $100^{\circ} \mathrm{C}$ for $10 \mathrm{~min}$. As an electron transport layer, a PCBM solution ( $20 \mathrm{mg} \mathrm{mL}^{-1}$ in chlorobenzene) was spin-coated at $2000 \mathrm{rpm}$ for $40 \mathrm{~s}$ and dried at $10{ }^{\circ} \mathrm{C}$ for $10 \mathrm{~min}$. For the formation of $\mathrm{FA}_{0.5} \mathrm{MA}_{0.5} \mathrm{~Pb}_{0.4} \mathrm{Sn}_{0.6} \mathrm{I}_{3}$ perovskite layer, a $\mathrm{Pb}_{0.4} \mathrm{Sn}_{0.6} \mathrm{I}_{2}$ composite film was deposited on the PCBM by coevaporation of $\mathrm{PbI}_{2}\left(0.8 \AA \mathrm{s}^{-1}\right)$ and $\mathrm{SnI}_{2}\left(1.2 \AA \mathrm{s}^{-1}\right.$, with $20 \mathrm{~mol} \%$ of $\mathrm{SnF}_{2}$ additive). The film was converted into $\mathrm{FA}_{0.5} \mathrm{MA}_{0.5} \mathrm{~Pb}_{0.4} \mathrm{Sn}_{0.6} \mathrm{I}_{3}$ by spin-coating $\mathrm{FA}_{0.5} \mathrm{MA}_{0.5} \mathrm{I}$ solution $\left(50 \mathrm{mg} \mathrm{mL}^{-1}\right.$ in IPA with $50 \mathrm{~mol} \%$ of thiourea additive) under $3500 \mathrm{rpm}$ for $45 \mathrm{~s}$ followed by an annealing process at $100^{\circ} \mathrm{C}$ for $10 \mathrm{~min}$. As a top hole-transport layer, $0.06 \mathrm{M}$ Spiro-OMeTAD dissolved in chlorobenzene $(28.8 \mu \mathrm{L}$ of 4 -tert-butylpyridine and $17.5 \mu \mathrm{L}$ of $1.8 \mathrm{M} \mathrm{Li}$-TFSI in acetonitrile) was spin-coated at $2000 \mathrm{rpm}$ for $20 \mathrm{~s}$. Finally, a gold electrode was deposited by thermal evaporation. The entire processes were conducted in a nitrogen-filled glove box.

Fabrication of the SPPD-OELG array. First, the patterned Au electrodes were thermally deposited onto a $\mathrm{SiO}_{2} / \mathrm{Si}$ substrate with a shadow mask. The organic and perovskite building blocks were fabricated onto the $\mathrm{Au}$ electrode in the following sequence with the same methods above: PEDOT:PSS/FA ${ }_{0.5} \mathrm{MA}_{0.5} \mathrm{~Pb}_{0.4} \mathrm{Sn}_{0.6} \mathrm{I}_{3}$ / $\mathrm{PCBM} / \mathrm{MAPbI}_{3} /$ Spiro-OMeTAD. A transparent ITO electrode was deposited by $\mathrm{RF}$ sputtering at $50 \mathrm{~W}$ under Ar gas at a pressure of $5 \mathrm{mTorr}$. To prevent physical damage to the underneath organic layer during the sputtering process, we deposited a $\mathrm{MoO}_{3}$ layer $(t \approx 30 \mathrm{~nm})$, serving as a buffer layer, on the SpiroOMeTAD using a thermal evaporator.

Material and device characterization. Cross-sectional TEM images of the SPPD were observed using Tecnai G2 F30 S-Twin microscope operated at an acceleration voltage of $300 \mathrm{kV}$. Cross-sectional samples $(t \approx 100 \mathrm{~nm})$ for the TEM measurement were obtained using focused-ion beam (FIB) system (HITACH NX5000). Elemental analyses of the perovskite films were performed by energy-dispersive spectrometer (EDS) mode of TEM with $136 \mathrm{eV}$ resolution and X-ray photoelectron spectroscopy (XPS, NEXSA) with $\mathrm{Al} \mathrm{Ka}$ source. Work function and binding energy of perovskite films on Au substrate were measured using ultraviolet photoelectron spectroscopy (UPS, NEXSA) with He (I) and He (II) source after Ar ion milling for $30 \mathrm{~s}$. The optical absorbance was measured using a UV-visible spectrometer (AvaSpec Spectrometer, Jinyoung Tech, Inc.). The optical images of perovskite photodetector array were observed by Olympus BX51 microscope. The photocurrent was measured using a Keithley 4200 source meter under illumination of 

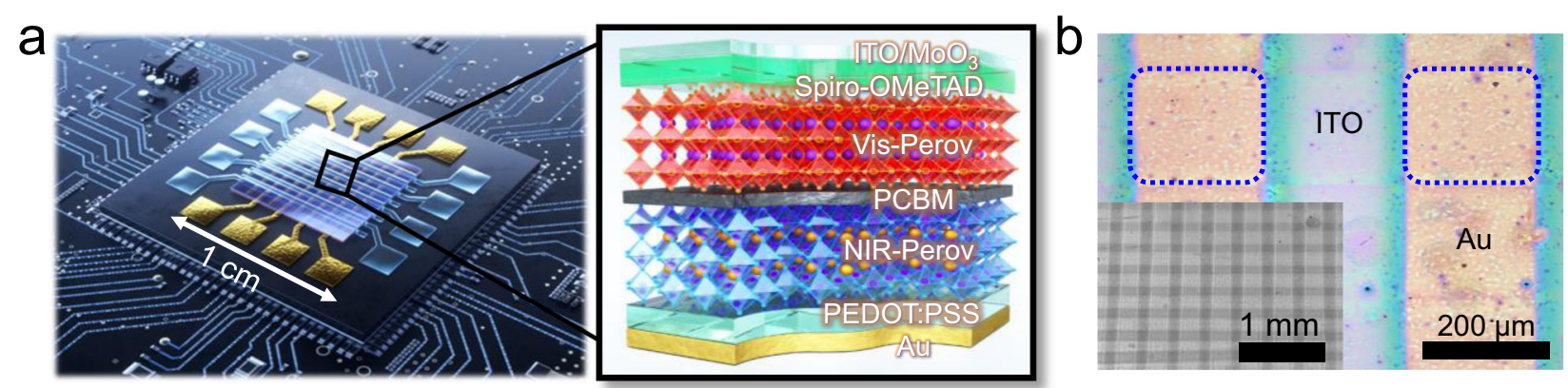

C

OR

00
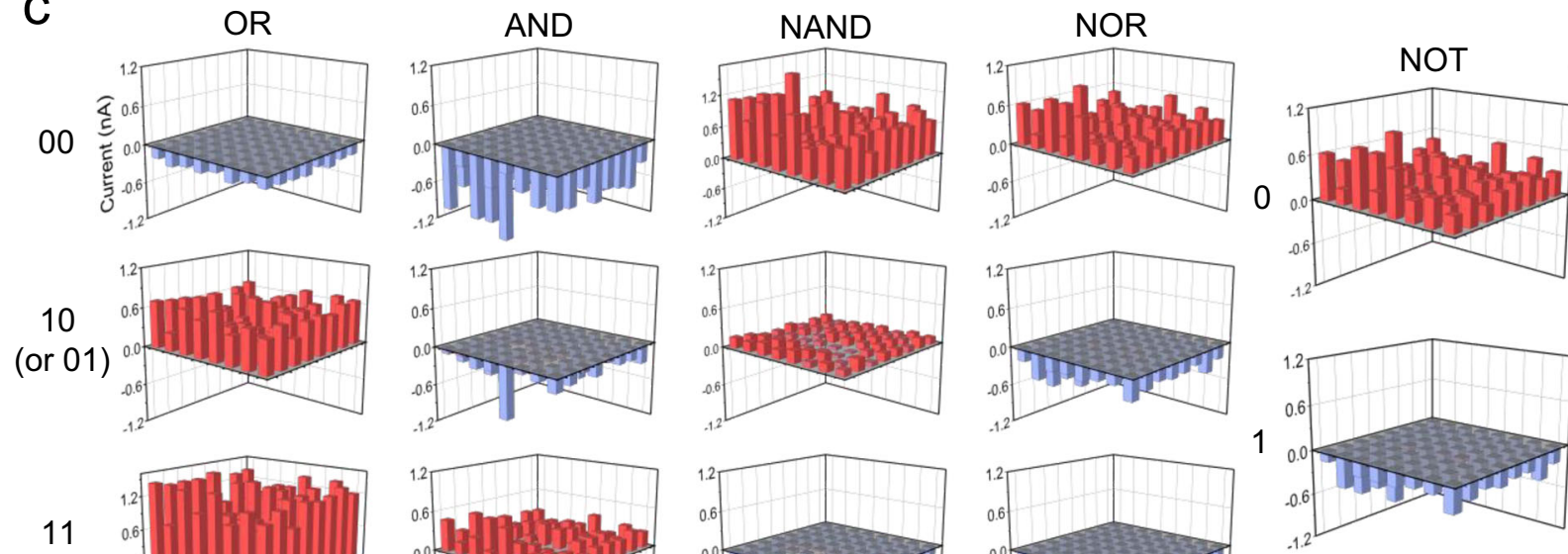

11
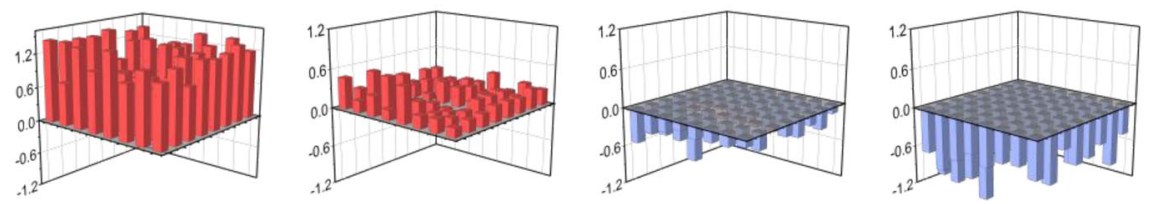

$0.2 \mathrm{nA}$

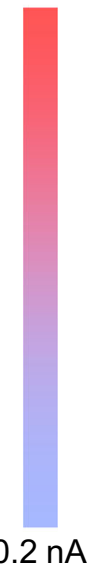

Fig. 4 Logic gate operation of the SPPD array. a Schematic of the ( $8 \times 8)$ crossbar-type SPPD array platform (left) and cross-sectional illustration (right) of a single SPPD. b Optical microscopy image of the SPPD array. The inset of $\mathbf{b}$ shows a low magnified image. $\mathbf{c}$ Three-dimensional bar charts for all the outputs ("OR", "AND", "NAND", "NOR", and "NOT") obtained from the 64 pixels. The red and blue bars show clear bipolar spectral photoresponses of all the pixels with reference to the fiducial level of $O \mathrm{nA}$ (gray face).

LEDs (530, 625, and $940 \mathrm{~nm}$, Mightex) and monochromatic light from xenon lamp (66902, Newport) equipped with monochromator (Mmac-200, Spectro). Rise and decay times were measured using an oscilloscope (Tektronix, DPD4014B) equipped with a low-noise current preamplifier (Stanford Research, SR570).

\section{Data availability}

The data that support the plots within this paper and other findings of this study are available from the corresponding author upon reasonable request.

Received: 15 April 2021; Accepted: 7 January 2022;

Published online: 07 February 2022

\section{References}

1. Caulfield, H. J. \& Dolev, S. Why future supercomputing requires optics. Nat. Photonics 4, 261-263 (2010).

2. Zhou, Z. \& Huang, D. Efficient and secure data storage operations for mobile cloud computing. in Proc. 2012 8th Int. Conf. Netw. Serv. Manag. CNSM 2012 37-45 (2012).

3. Mellit, A. \& Kalogirou, S. A. MPPT-based artificial intelligence techniques for photovoltaic systems and its implementation into field programmable gate array chips: Review of current status and future perspectives. Energy 70, 1-21 (2014).

4. Hassan, S. \& Asghar, M. Limitation of silicon based computation and future prospects. in 2010 Second International Conference on Communication Software and Networks. 559-561 (IEEE, 2010).

5. Avouris, P. \& Chen, J. Nanotube electronics and optoelectronics. Mater. Today 9, 46-54 (2006).

6. Kim, J. H. et al. All-optical AND gate using cross-gain modulation in semiconductor optical amplifiers. Jpn. J. Appl. Phys. 43, 608 (2004).
7. Minzioni, P., Lacava, C., Tanabe, T. \& Dong, J. Roadmap on all-optical processing. J. Opt. 21, 063001 (2019).

8. Kim, S. H. et al. All-optical half adder using cross gain modulation in semiconductor optical amplifiers. Opt. Express 14, 10693-10698 (2006).

9. Taraphdar, C., Chattopadhyay, T. \& Roy, J. N. Mach-Zehnder interferometer-based all-optical reversible logic gate. Opt. Laser Technol. 42, 249-259 (2010).

10. Singh, P., Tripathi, D. K., Jaiswal, S. \& Dixit, H. K. All-optical logic gates: designs, classfication, and comparison. Adv. Opt. Technol. 275083, https:// doi.org/10.1155/2014/275083 (2014).

11. Zeng, S., Zhang, Y., Li, B. \& Pun, E. Y.-B. Ultrasmall optical logic gates based on silicon periodic dielectric waveguides. Photonics Nanostruct.: Fundam. Appl. 8, 32-37 (2010).

12. Fetterman, M. R. Design for high-speed optoelectronic boolean logic. IEEE Photonics Technol. Lett. 21, 1740-1742 (2009).

13. Chen, H., Liu, H., Zhang, Z., Hu, K. \& Fang, X. Nanostructured photodetectors: from ultraviolet to terahertz. Adv. Mater. 28, 403-433 (2016).

14. Kim, J. et al. Photon-triggered nanowire transistors. Nat. Nanotechnol. 12, 963-968 (2017).

15. Yang, C. M. et al. Bidirectional all-optical synapses based on a $2 \mathrm{D} \mathrm{Bi}_{2} \mathrm{O}_{2} \mathrm{Se} /$ graphene hybrid structure for multifunctional optoelectronics. Adv. Funct. Mater. 30, 1-12 (2020).

16. Kim, B. J. et al. Highly transparent phototransistor based on quantum-dots and $\mathrm{ZnO}$ bilayers for optical logic gate operation in visible-light. RSC Adv. 10, 16404-16414 (2020).

17. $\mathrm{Li}, \mathrm{M}$. et al. The fabrication of a self-powered $\mathrm{CuInS}_{2} / \mathrm{TiO}_{2}$ heterojunction photodetector and its application in visible light communication with ultraviolet light encryption. J. Mater. Chem. C 9, 14613-14622 (2021).

18. Liu, Y. et al. High performance $\mathrm{MoO}_{3-\mathrm{x}} / \mathrm{Si}$ heterojunction photodetectors with nanoporous pyramid $\mathrm{Si}$ arrays for visible light communication application. $J$ Mater. Chem. C. 7, 917-925 (2019).

19. Quyang, B. et al. Dual-polarity output response-based photoelectric devices. Cell Rep. Phys. Sci. 2, 100418 (2021). 
20. Quyang, B. et al. Dual-polarity response in self-powered $\mathrm{ZnO} \mathrm{NWs} / \mathrm{Sb}_{2} \mathrm{Se}_{3}$ film heterojunction photodetector array for optical communication. Nano Energy 68, 104312 (2020).

21. Quyang, B. et al. Photocurrent polarity controlled by light wavelength in selfpowered $\mathrm{ZnO}$ nanowires/SnS photodetector system. iScience 1, 16-23 (2018).

22. Yang, Y. Controlling photocurrent direction with light. Nat. Electron. 4, 631-632 (2021).

23. Huang, Y. et al. Logic gates and computation from assembled nanowire building blocks. Science 294, 1313-1317 (2001).

24. Kim, D. R., Lee, C. H. \& Zheng, X. Direct growth of nanowire logic gates and photovoltaic devices. Nano Lett. 10, 1050-1054 (2010).

25. Ball, J. M., Lee, M. M., Hey, A. \& Snaith, H. J. Low-temperature processed meso-superstructured to thin-film perovskite solar cells. Energy Environ. Sci. 6, 1739-1743 (2013)

26. Miyata, A. et al. Direct measurement of the exciton binding energy and effective masses for charge carriers in organic-inorganic tri-halide perovskites. Nat. Phys. 11, 582-587 (2015).

27. Park, N. G. Organometal perovskite light absorbers toward a $20 \%$ efficiency lowcost solid-state mesoscopic solar cell. J. Phys. Chem. Lett. 4, 2423-2429 (2013).

28. Saliba, M., Correa-Baena, J. P., Grätzel, M., Hagfeldt, A. \& Abate, A. Perovskite solar cells: from the atomic level to film quality and device performance. Angew. Chem. - Int. Ed. 57, 2554-2569 (2018).

29. Zhang, M. et al. High-performance photodiode-type photodetectors based on polycrystalline formamidinium lead iodide perovskite thin films. Sci. Rep. 8, 1-9 (2018).

30. Li, J. et al. Self-trapped state enabled filterless narrowband photodetections in 2D layered perovskite single crystals. Nat. Commun. 10, 1-10 (2019).

31. Liu, D. et al. Self-powered and broadband lead-free inorganic perovskite photodetector with high stability. ACS Appl. Mater. Interfaces 12, 30530-30537 (2020).

32. Yu, J. et al. A high-performance self-powered broadband photodetector based on a $\mathrm{CH}_{3} \mathrm{NH}_{3} \mathrm{PbI}_{3}$ perovskite/ZnO nanorod array heterostructure. J. Mater. Chem. C. 4, 7302-7308 (2016)

33. Leung, S. F. et al. A self-powered and flexible organometallic halide perovskite photodetector with very high detectivity. Adv. Mater. 30, 1-8 (2018).

34. Alwadai, N. et al. High-performance ultraviolet-to-infrared broadband perovskite photodetectors achieved via inter-/intraband transitions. ACS Appl. Mater. Interfaces 9, 37832-37838 (2017).

35. Guan, X. et al. A monolithic artificial iconic memory based on highly stable perovskite-metal multilayers. Appl. Phys. Rev. 7, 031401 (2020).

36. Lin, C.-H. et al. Orthogonal lithography for halide perovskite optoelectronic nanodevices. ACS Nano 13, 1168-1176 (2018).

37. Kim, H. et al. Bias-modulated multicolor discrimination enabled by an organic-inorganic hybrid perovskite photodetector with a $\mathrm{p}-\mathrm{i}-\mathrm{n}-\mathrm{i}-\mathrm{p}$ configuration. Laser Phtonics Rev. 14, 2000305 (2020).

38. Hu, H., Dong, B. \& Zhang, W. Low-toxic metal halide perovskites: opportunities and future challenges. J. Mater. Chem. A 5, 11436-11449 (2017).

39. Liao, W. et al. Fabrication of efficient low-bandgap perovskite solar cells by combining formamidinium tin iodide with methylammonium lead iodide. $J$. Am. Chem. Soc. 138, 12360-12363 (2016).

40. Zhao, J., Li, N., Cui, G. \& Zhao, J. Study on immersion tin process by electrochemical methods and molecular orbital theory. J. Electrochem. Soc. 153, C848-C853 (2006).

41. Dong, $\mathrm{H}$. et al. Improving electron extraction ability and device stability of perovskite solar cells using a compatible PCBM/AZO electron transporting bilayer. Nanomaterials 8, 720 (2018).

42. Li, Z. et al. A facilely synthesized 'spiro' hole-transporting material based on spiro[3.3] heptane-2,6-dispirofluorene for efficient planar perovskite solar cells. RSC Adv. 7, 41903-41908 (2017).

43. Ricciarelli, D. et al. Instability of tin iodide perovskites: bulk p-doping versus tin oxidation. ACS Energy Lett. 5, 2787-2795 (2020).
44. Burkhard, G. F., Hoke, E. T. \& McGehee, M. D. Accounting for interference, scattering, and electrode absorption to make accurate internal quantum efficiency measurements in organic and other thin solar cells. Adv. Mater. 22, 3293-3297 (2010)

45. $\mathrm{Wu}, \mathrm{P}$. et al. Two-dimensional transistors with reconfigurable polarities for secure circuits. Nat. Electron. 4, 45-53 (2021).

46. Khan, Y. et al. A flexible organic reflectance oximeter array. PNAS 115 E11015-E11024 (2018).

\section{Acknowledgements}

W.K., G.Y.J., and Y.P. acknowledge the National Research Foundation of Korea (NRF) grant funded by the Korea government (MIST and MOE) (No. NRF-2019R1A2B5B01070640, 2020M3H5A108110412, 2019M3E7A1113097) and the KIST Institutional grant (2E31271). A.A.S. acknowledges the CSC-IT Center for Science (Finland) for the computational resources.

\section{Author contributions}

W.K. and H.K. contributed equally to this work. W.K. and H.K. conceived the idea, carried out the experiments, and wrote the manuscript. T.J.Y., J.Y.L., B.H.L., and J.Y.J contributed to the discussion on device fabrication and characterization. A.A.S. contributed to the analytical and computational analysis. G.Y.J. and Y.P. supervised and coordinated the entire works. All authors discussed the final manuscript, commented, and revised the submitted manuscript.

\section{Competing interests}

The authors declare no competing interests.

\section{Additional information}

Supplementary information The online version contains supplementary material available at https://doi.org/10.1038/s41467-022-28374-w.

Correspondence and requests for materials should be addressed to Gun Young Jung or Yusin Pak.

Peer review information Nature Communications thanks Ya Yang and the other, anonymous, reviewer(s) for their contribution to the peer review of this work. Peer reviewer reports are available.

Reprints and permission information is available at http://www.nature.com/reprints

Publisher's note Springer Nature remains neutral with regard to jurisdictional claims in published maps and institutional affiliations.

Open Access This article is licensed under a Creative Commons Attribution 4.0 International License, which permits use, sharing, adaptation, distribution and reproduction in any medium or format, as long as you give appropriate credit to the original author(s) and the source, provide a link to the Creative Commons license, and indicate if changes were made. The images or other third party material in this article are included in the article's Creative Commons license, unless indicated otherwise in a credit line to the material. If material is not included in the article's Creative Commons license and your intended use is not permitted by statutory regulation or exceeds the permitted use, you will need to obtain permission directly from the copyright holder. To view a copy of this license, visit http://creativecommons.org/ licenses/by/4.0/.

(C) The Author(s) 2022 